No. 572

June 2017

Natural Convection Flow of a Two-Phase

Dusty Non-Newtonian Fluid Along a Vertical Surface

S. Siddiqa, N. Begum,

Md. A. Hossain, R. S. R. Gorla 


\title{
Natural Convection Flow of a Two-Phase Dusty Non-Newtonian Fluid Along a Vertical Surface
}

\author{
Sadia Siddiqa*,1, Naheed Begum ${ }^{\S}$, Md. Anwar Hossain ${ }^{\ddagger}$, Rama Subba Reddy Gorla ${ }^{\dagger}$ \\ * Department of Mathematics, COMSATS Institute of Information Technology, Kamra \\ Road, Attock, Pakistan \\ $\S$ Institute of Applied Mathematics (LSIII), TU Dortmund, Vogelpothsweg 87, D-44221 \\ Dortmund, Germany \\ $\ddagger$ UGC Professor, University of Dhaka, Dhaka, Bangladesh \\ ${ }^{\dagger}$ Department of Mechanical \& Civil Engineering, Purdue University Northwest, Westville, \\ IN 46391
}

\begin{abstract}
The aim of this paper is to present a boundary-layer analysis of two-phase dusty non-Newtonian fluid flow along a vertical surface by using a modified power-law viscosity model. This investigation particularly reports the flow behavior of spherical particles suspended in the non-Newtonian fluid. The governing equations are transformed into nonconserved form and then solved straightforwardly by implicit finite difference method. The numerical results of rate of heat transfer, rate of shear stress, velocity and temperature profiles and streamlines and isotherms are presented for wide range of Prandtl number, i.e, $(0.7 \leq \operatorname{Pr} \leq 1000.0)$, with the representative values of the power-law index $n$. A good agreement is found between the present and the previous results when compared with some special cases. The key observation from the present study is that the power-law fluids with $(n>1)$ are more likely to promote the rate of heat transfer near the leading edge.
\end{abstract}

Keywords: Natural Convection, Dusty Fluid, Two-Phase, Non-Newtonian Fluids, Modified Power Law, Vertical Surface

\section{Nomenclature}

$C_{f} \quad$ Skin friction coefficient

$c_{p} \quad$ Specific heat at constant pressure for fluid-phase

$c_{s} \quad$ Specific heat at constant pressure for particle-phase

$D_{\rho} \quad$ Mass concentration parameter

$g \quad$ Acceleration due to gravity

Gr Generalized Grashof number

$K$ Dimensional empirical constant appeared in power-law

$L \quad$ Characteristic length

$n \quad$ Power-law index

$N u \quad$ Nusselt number coefficient

$\hat{p} \quad$ Dimensional pressure of carrier phase

$\hat{p}_{p} \quad$ Dimensional pressure of particle phase

$p \quad$ Dimensionless pressure of the carrier phase

$p_{p} \quad$ Dimensionless pressure of the particle phase

Pr Prandtl number

\footnotetext{
${ }^{1}$ Corresponding author.

Email: saadiasiddiqa@gmil.com
} 
$Q \quad$ Rate of heat transfer at the surface

$T$ Dimensional temperature of fluid-phase

$T_{w} \quad$ Surface temperature

$T_{\infty} \quad$ Ambient fluid temperature

$T_{p} \quad$ Dimensional temperature of particle-phase

$\Delta T$ Difference between surface temperature and ambient fluid temperature

$\hat{u}, \hat{v} \quad$ Dimensional fluid-phase velocity components

$\hat{u}_{p}, \hat{v}_{p}$ Dimensional particle-phase velocity components

$u, v \quad$ Dimensionless fluid-phase velocity components

$u_{p}, v_{p}$ Dimensionless particle-phase velocity components

$\bar{x}, \bar{y}$ Dimensional cartesian coordinates

$x, y \quad$ Dimensionless coordinate system

$U_{c} \quad$ Reference velocity

Greek letters

$\alpha \quad$ Thermal diffusivity

$\alpha_{d} \quad$ Dusty fluid parameter

$\beta \quad$ Volumetric expansion coefficient

$\gamma \quad$ Ratio of $c_{p}$ to $c_{s}$

$\kappa \quad$ Thermal conductivity

$\theta \quad$ Dimensionless fluid-phase temperature

$\theta_{p} \quad$ Dimensionless particle-phase temperature

$\rho \quad$ Density of fluid-phase

$\rho_{p} \quad$ Density of particle-phase

$\mu \quad$ Dynamic viscosity of fluid

$\nu \quad$ Kinematic viscosity of fluid

$\tau_{m} \quad$ Velocity relaxation time of the particles

$\tau_{T} \quad$ Thermal relaxation time of the particles

$\tau_{w} \quad$ Shear stress at the surface

\section{Subscripts}

$w \quad$ surface condition

$\infty \quad$ ambient condition

$p \quad$ particle phase

Superscripts

- dimensional system

$(i, j)$ nodal positions

\section{Introduction}

The interest in studying the dynamics of heat transfer problems involving non-Newtonian power-law fluids has been widely increased in the past half century, because of their wide range of usage in processing and manufacturing industries. For instance, most of the par- 
ticulate slurries such as coal in water, synthetic lubricants, polymers, paints, emulsions, biological fluids such as blood, food stuffs such as jams, jellies and marmalades are few examples of fluids exhibit the non-Newtonian behavior. Although, several number of constitutive laws have been established to describe the behavior of non-Newtonian fluids, but the most deliberately used model in non-Newtonian fluid mechanics is the Ostwald-de Waele type power-law model (implied by [1]). Numerous researchers studied heat and mass transfer by taking taking into account power-law fluids. In this regard, Schowalter [2] applied the boundary-layer theory to shear thinning fluids (fluids for which power-law index is less than 1). After that Lee and Ames [3] extended the work of Schowalter [2] and established the similar solutions for power-law fluids. A theoretical analysis of laminar natural convection heat transfer to non-Newtonian fluids was conducted by Acrivos [4]. In that paper, the author investigated how the well-established expressions for the rate of heat transfer of Newtonian fluids can be generalized to include the non-Newtonian effects. A complete survey of the literature on non-Newtonian fluids is impractical however a few items are listed here to provide starting points for a broader literature (for details see Refs. [5]-[9]). In later years, Kawase and Ulbrecht [10] presented the approximate solution to the natural convection heat transfer from a vertical plate. Afterwards, Huang et al. [11] reported the influence of Prandtl number on free convection flow of power-law non-Newtonian fluids from a vertical plate. In [11], the author presented similarity solutions and concluded that the average rate of heat transfer increase when Prandtl number rises. Later on, Kumari et al. [12] presented a theoretical analysis for laminar natural convection boundary layer flow of non-Newtonian power-law fluid. In that paper, the authors considered the vertical sinusoidal wavy geometry and established the numerical solutions via Keller-Box method for wide range of Prandtl number. Subsequently, a large amount of work for non-Newtonian fluids including integral, experimental, and numerical methods, was presented under various physical circumstances (see Refs. [13]-[17]) .

In all above-mentioned studies, attention has been given to fluids which are free from all impurities (clear fluid). But, pure fluid is rarely available in many practical situations, for instance, common fluids like air and water contains impurities like dust particles. Therefore, the analysis of the flow of fluids with suspended particles or gas-particle mixture have received notable attention due to its practical applications in atmospheric, engineering and physiological fields. Solid rocket exhaust nozzles, combustion chambers, blast waves moving over the Earth's surface, conveying of powdered materials, fluidized beds, environmental pollutants, petroleum industry, purification of crude oil and other technological fields are some of the practical applications of dusty fluids (see [18]). In this regard, Farbar and Morley [19] were the first to analyze the gas-particulate suspension on experimental grounds. After that, Marble [20] studied the problem of dynamics of a gas containing small solid particles and developed the equations for gas-particle flow systems. Singleton [21] was the first to study the boundary layer analysis for dusty fluid and later on several attempts were made to conclude the physical insight of such two-phase flows (see Refs. [22]-[30]) under different physical circumstances. In addition, Siddiqa et al. [32] reported the influence of thermal radiation on natural convection flow of contaminated air and water along the vertical wavy frustum of a cone. Very recently, the problem of compressible dusty gas along a vertical wavy surface was investigated numerically by Siddiqa et al. [33]. In that article, the authors solved the physical model numerically and reported the effect of compressibility, particulate suspension and sinusoidal waveform on rate of heat transfer and the flow characteristics. 
It is observed that most of the research related to two-phase (particle-fluid) flow assumes the fluid to be Newtonian in nature because such fluids have linear relationship between the shear stress and the shear rate. The applications of non-Newtonian power-law dusty fluids are found in process engineering, therefore it becomes important to reveal their flow characteristics. In this regard, Chamkha [34] studied the unsteady flow of a power-law dusty fluid with suction but the author did not considered the heat transfer phenomena. Thus, present work has been undertaken to give the more detailed analysis of the natural convection flow of a power-law dusty fluid by considering the thermal energy phenomena. The equations that govern the two phase flow are reduced to a dimensionless form and then the coordinate transformation (primitive variable formulation) is employed to transform the two-phase boundary layer model into a convenient form. Since the equations are coupled and nonlinear, the solutions are obtained numerically by applying two point implicit finite difference method. The results for the two-phase problem are displayed in the form of wall shear stress, heat transfer rate, velocity and temperature profiles, streamlines and isotherms by varying several controlling parameters. The computational results for carrier phase are also compared with published data of various studies and all agrees well with the present solutions. The effects of the presence of dust particle and the non-Newtonian nature of the fluids on flow and heat transfer characteristics are examined and discussed in detail. For full demonstration of the various non-Newtonian fluids, the behaviors of both Newtonian and dilatant fluids on the natural convection laminar flow along a vertical heated wall are studied by choosing the power-law index as $n=1.0,1.2,1.5,1.8,2.0$.

\section{Formulation of the Problem}

The physical model considered here is an isothermal vertical wall with a temperature, $T_{w}$, which is situated in two-phase dusty power-law fluid with ambient temperature, $T_{\infty}$, such that $T_{w}>T_{\infty}$. In our detail computational work, the kinematic viscosity $\nu$ depends on shear-rate and is correlated by a modified power-law. Under the assumptions of two-phase flow given in [30] and [32], the governing equations for non-Newtonian, steady, laminar and incompressible fluid are given by (see Refs. [21], [23]):

For the fluid phase:

$$
\begin{gathered}
\frac{\partial \hat{u}}{\partial \hat{x}}+\frac{\partial \hat{v}}{\partial \hat{y}}=0 \\
\rho\left(\hat{u} \frac{\partial \hat{u}}{\partial \hat{x}}+\hat{v} \frac{\partial \hat{u}}{\partial \hat{y}}\right)=-\frac{\partial \hat{p}}{\partial \hat{x}}+\frac{\partial}{\partial \hat{y}}\left(\mu \frac{\partial \hat{u}}{\partial \hat{y}}\right)+\rho g \beta\left(T-T_{\infty}\right)+\frac{\rho_{p}}{\tau_{m}}\left(\hat{u}_{p}-\hat{u}\right) \\
\rho\left(\hat{u} \frac{\partial \hat{v}}{\partial \hat{x}}+\hat{v} \frac{\partial \hat{v}}{\partial \hat{y}}\right)=-\frac{\partial \hat{p}}{\partial \hat{y}}+\frac{\partial}{\partial \hat{y}}\left(\mu \frac{\partial \hat{v}}{\partial \hat{y}}\right)+\frac{\rho_{p}}{\tau_{m}}\left(\hat{v}_{p}-\hat{v}\right) \\
\rho c_{p}\left(\hat{u} \frac{\partial T}{\partial \hat{x}}+\hat{v} \frac{\partial T}{\partial \hat{y}}\right)=\kappa \frac{\partial^{2} T}{\partial \hat{y}^{2}}+\frac{\rho_{p} c_{s}}{\tau_{T}}\left(T_{p}-T\right)
\end{gathered}
$$

For the particle phase:

$$
\begin{gathered}
\frac{\partial \hat{u}_{p}}{\partial \hat{x}}+\frac{\partial \hat{v}_{p}}{\partial \hat{y}}=0 \\
\rho_{p}\left(\hat{u}_{p} \frac{\partial \hat{u}_{p}}{\partial \hat{x}}+\hat{v}_{p} \frac{\partial \hat{u}_{p}}{\partial \hat{y}}\right)=-\frac{\partial \hat{p}_{p}}{\partial \hat{x}}-\frac{\rho_{p}}{\tau_{m}}\left(\hat{u}_{p}-\hat{u}\right)
\end{gathered}
$$




$$
\begin{gathered}
\rho_{p}\left(\hat{u}_{p} \frac{\partial \hat{v}_{p}}{\partial \hat{x}}+\hat{v}_{p} \frac{\partial \hat{v}_{p}}{\partial \hat{y}}\right)=-\frac{\partial \hat{p}_{p}}{\partial \hat{y}}-\frac{\rho_{p}}{\tau_{m}}\left(\hat{v}_{p}-\hat{v}\right) \\
\rho_{p} c_{s}\left(\hat{u}_{p} \frac{\partial T_{p}}{\partial \hat{x}}+\hat{v}_{p} \frac{\partial T_{p}}{\partial \hat{y}}\right)=-\frac{\rho_{p} c_{s}}{\tau_{T}}\left(T_{p}-T\right)
\end{gathered}
$$

where $(\hat{u}, \hat{v}), T, \hat{p}, \rho, c_{p}, \beta, \kappa, \nu$ are respectively the velocity vector in the $(\hat{x}, \hat{y})$ direction, temperature, pressure, density, specific heat at constant pressure, volumetric expansion coefficient, thermal conductivity and kinematic viscosity of the fluid/carrier phase. Similarly, $\left(\hat{u}_{p}, \hat{v}_{p}\right), T_{p}, \hat{p}_{p}, \rho_{p}$ and $c_{s}$ corresponds to the velocity vector, temperature, pressure, density and specific heat for the particle phase. Further, $g$ is the acceleration due to gravity and $\tau_{m}$ $\left(\tau_{T}\right)$ is the momentum relaxation time (thermal relaxation time) during which the velocity (temperature) of the particle phase relative to the fluid is reduced to $1 / e$ times its initial value. It can be noted that in the above equations the term $\rho_{p}\left(\hat{u}_{p}-\hat{u}\right) / \tau_{m}$ is the force per unit volume of mixture of both phases acting on the fluid or the net effect of the dust on the fluid particles. Moreover, $\rho_{p} c_{s}\left(T_{p}-T\right) / \tau_{T}$ is the total heat transfer to the fluid per unit volume of the mixture of the two phases. The viscosity is correlated by a modified power-law (for details see Ref. [1]), defined as:

$$
\nu=\frac{K}{\rho}\left|\frac{\partial \hat{u}}{\partial \hat{y}}\right|^{n-1}
$$

where $K$ and $n$ are empirical constants characteristic of the fluid. It is important to mention here that, i) for $n<1$ the class of fluids is pseudo-plastic (shear thinning), ii) for $n>1$ the fluid is dilatant (shear thickening) and iii) for $n=1$ the fluids are simply of Newtonian type.

The fundamental equations stated above are to be solved under appropriate boundary conditions to determine the flow fields of the fluid and the dust particles. Therefore, boundary conditions for the fluid phase are:

$$
\begin{gathered}
\hat{u}(\hat{x}, 0)=\hat{v}(\hat{x}, 0)=T(\hat{x}, 0)-T_{w}=0 \\
u(\hat{x}, \infty)=T(\hat{x}, \infty)-T_{\infty}=0
\end{gathered}
$$

Boundary conditions for the particle phase are:

$$
\begin{gathered}
\hat{u}_{p}(\hat{x}, 0)=\hat{v}_{p}(\hat{x}, 0)=T_{p}(\hat{x}, 0)-T_{w}=0 \\
\hat{u}_{p}(\hat{x}, \infty)=T_{p}(\hat{x}, \infty)-T_{\infty}=0
\end{gathered}
$$

Following dimensionless variables are now introduced for non-dimensionalization of the governing equations of two-phase model:

$$
\begin{array}{r}
x=\frac{\hat{x}}{L}, \quad y=\frac{\hat{y}}{L} G r^{1 / 2(n+1)}, \quad\left(u, u_{p}\right)=\frac{\left(\hat{u}, \hat{u}_{p}\right)}{U_{c}}, \quad\left(v, v_{p}\right)=\frac{\left(\hat{v}, \hat{v}_{p}\right)}{U_{c}} G r^{1 / 2(n+1)}, \\
U_{c}=(g \beta \triangle T L)^{1 / 2}, \quad\left(p, p_{p}\right)=\frac{\left(\hat{p}, \hat{p}_{p}\right)}{\rho U_{c}^{2}}, \quad\left(\Theta, \Theta_{p}\right)=\frac{\left(T, T_{p}\right)-T_{\infty}}{\triangle T}, \quad \triangle T=T_{w}-T_{\infty},
\end{array}
$$

where $G r=\left((g \beta \triangle T)^{2-n} L^{2+n}\right) /(K / \rho)^{2}$ is the generalized Grashof number. With the help of (12) governing equations (1)-(11) becomes:

For the fluid phase:

$$
\frac{\partial u}{\partial x}+\frac{\partial v}{\partial y}=0
$$




$$
\begin{gathered}
u \frac{\partial u}{\partial x}+v \frac{\partial u}{\partial y}=-\frac{\partial p}{\partial x}+\frac{\partial}{\partial y}\left(\frac{\partial u}{\partial y}\left|\frac{\partial u}{\partial y}\right|^{n-1}\right)+\theta+D_{\rho} \alpha_{d}\left(u_{p}-u\right) \\
\frac{\partial p}{\partial y}=0 \\
u \frac{\partial \theta}{\partial x}+v \frac{\partial \theta}{\partial y}=\frac{1}{\operatorname{Pr}}\left(\frac{\partial^{2} \theta}{\partial y^{2}}+\frac{2}{3} D_{\rho} \alpha_{d}\left(\theta_{p}-\theta\right)\right)
\end{gathered}
$$

For the particle phase:

$$
\begin{gathered}
\frac{\partial u_{p}}{\partial x}+\frac{\partial v_{p}}{\partial y}=0 \\
u_{p} \frac{\partial u_{p}}{\partial x}+v_{p} \frac{\partial u_{p}}{\partial y}=-\frac{\partial p_{p}}{\partial x}-\alpha_{d}\left(u_{p}-u\right) \\
\frac{\partial p_{p}}{\partial y}=0 \\
u_{p} \frac{\partial \theta_{p}}{\partial x}+v_{p} \frac{\partial \theta_{p}}{\partial y}=-\frac{2}{3 \gamma \operatorname{Pr}} \alpha_{d}\left(\theta_{p}-\theta\right)
\end{gathered}
$$

where

$\gamma=\frac{c_{s}}{c_{p}}, \tau_{T}=\frac{3}{2} \gamma \tau_{m} \operatorname{Pr}, D_{\rho}=\frac{\rho_{p}}{\rho}, \alpha_{d}=\frac{1}{\tau_{m}}\left(\frac{L}{g \beta \triangle T}\right)^{1 / 2}, \operatorname{Pr}=\frac{1}{\alpha}\left(\frac{K}{\rho}\right)^{\frac{2}{(1+n)}} L^{\frac{(1-n)}{(1+n)}} U_{c}^{\frac{3(n-1)}{(n+1)}}$

It is important to mention here that for different mixtures, the interaction term $\gamma$ may vary between 0.1 and 10.0 (For details see [18]). It can also be observed that for $\alpha_{d}=0.0$, the flow represents the problem of natural convection in the absence of the dust particles (i.e carrier phase only). In the present problem we have considered that the lower order pressure gradient along the $x$-direction is determined from the inviscid solution (from Eqs. (14) and (18), therefore $\partial p / \partial x=\partial p_{p} / \partial x=0$. The boundary conditions becomes:

$$
\begin{gathered}
u(x, 0)=v(x, 0)=\theta(x, 0)-1=0 \\
u(x, \infty)=\theta(x, \infty)=0 \\
u_{p}(x, 0)=v_{p}(x, 0)=\theta_{p}(x, 0)-1=0 \\
u_{p}(x, \infty)=\theta_{p}(x, \infty)=0
\end{gathered}
$$

Now, we propose to integrate the above system of equations numerically for two-phase model. The numerical scheme is applied after converting the dimensionless equations into a suitable form with the help of primitive variable formulations. For this, we use the following set of transformations:

$$
\begin{array}{r}
x=X, \quad y=(4 x)^{n / 2(n+1)} Y, \quad\left(u, u_{p}\right)=(4 x)^{1 / 2}\left(U, U_{p}\right), \quad\left(v, v_{p}\right)=(4 x)^{-1 / 2(n+1)}\left(V, V_{p}\right), \\
\left(\theta, \theta_{p}\right)=\left(\Theta, \Theta_{p}\right)
\end{array}
$$

By using (23), the equations (13)-(20) subject to the boundary conditions (22) will be mapped into the following system of partial differential equations:

For the fluid phase:

$$
2 U+(4 X) \frac{\partial U}{\partial X}-\frac{2 n}{(n+1)} Y \frac{\partial U}{\partial Y}+\frac{\partial V}{\partial Y}=0
$$




$$
\begin{array}{r}
2 U^{2}+(4 X) U \frac{\partial U}{\partial X}+\left(V-\frac{2 n}{(n+1)} Y U\right) \frac{\partial U}{\partial Y}=(4 X)^{(1-n) / 2(n+1)} \frac{\partial}{\partial Y}\left(\frac{\partial U}{\partial Y}\left|\frac{\partial U}{\partial Y}\right|^{n-1}\right)+\Theta \\
+D_{\rho} \alpha_{d}(4 X)^{1 / 2}\left(U_{p}-U\right)
\end{array}
$$

$$
\begin{aligned}
(4 X) U \frac{\partial \Theta}{\partial X}+\left(V-\frac{2 n}{(n+1)} Y U\right) \frac{\partial \Theta}{\partial Y} & =\frac{1}{\operatorname{Pr}}(4 X)^{(1-n) / 2(n+1)} \frac{\partial^{2} \Theta}{\partial Y^{2}} \\
+ & \frac{2}{3 \operatorname{Pr}} D_{\rho} \alpha_{d}(4 X)^{1 / 2}\left(\Theta_{p}-\Theta\right)
\end{aligned}
$$

For the particle phase:

$$
\begin{gathered}
2 U_{p}+(4 X) \frac{\partial U_{p}}{\partial X}-\frac{2 n}{(n+1)} Y \frac{\partial U_{p}}{\partial Y}+\frac{\partial V_{p}}{\partial Y}=0 \\
2 U_{p}^{2}+(4 X) U_{p} \frac{\partial U_{p}}{\partial X}+\left(V_{p}-\frac{2 n}{(n+1)} Y U_{p}\right) \frac{\partial U_{p}}{\partial Y}=-\alpha_{d}(4 X)^{1 / 2}\left(U_{p}-U\right) \\
(4 X) U_{p} \frac{\partial \Theta_{p}}{\partial X}+\left(V_{p}-\frac{2 n}{(n+1)} Y U_{p}\right) \frac{\partial \Theta_{p}}{\partial Y}=-\frac{2}{3 \gamma \operatorname{Pr}} \alpha_{d}(4 X)^{1 / 2}\left(\Theta_{p}-\Theta\right)
\end{gathered}
$$

The boundary conditions becomes:

$$
\begin{gathered}
U(X, 0)=V(X, 0)=\Theta(X, 0)-1=0 \\
U(X, \infty)=\Theta(X, \infty)=0 \\
U_{p}(X, 0)=V_{p}(X, 0)=\Theta_{p}(X, 0)-1=0 \\
U_{p}(X, \infty)=\Theta_{p}(X, \infty)=0
\end{gathered}
$$

\section{Solution Methodology}

The continuity, momentum and energy equations of carrier and disperse phase, given in (24) to (29) subject to the boundary conditions (30) are solved numerically with the aid of the implicit finite difference method which implies Thomas algorithm as a solver. Since the equations are parabolic in $X$ therefore solutions can be marched in the downstream direction. The computational domain is discretized over the entire boundary layer region. Keeping numerical stability in view, two-point central difference and backward difference quotients are respectively used for diffusion and convective terms. The resulting system of algebraic equations can be cast into a tri-diagonal matrix equation which is solved via Thomas algorithm. This algorithm works on the following pattern (For details see Ref. [35]):

1. Set the suitable initial and boundary conditions.

2. Solve the unknowns $U, U_{p}, \Theta$ and $\Theta_{p}$ at $Y=0$. It implies that these unknown quantities meet the convergence criteria.

3. Solve for the next step $Y_{j}=Y_{j-1}+\Delta Y$ by using the solution position.

4. The computations are iterated until the following convergence criteria is achieved:

$$
\left(\max \left|U_{i, j}\right|+\max \left|U_{p} i, j\right|+\max \left|V_{i, j}\right|+\max \left|V_{p} i, j\right|+\max \left|\Theta_{i, j}\right|+\max \left|\Theta_{p} i, j\right|\right) \leq 10^{-6}
$$




\section{Repeat step 2-4 for $X$ maximum.}

In the computation procedure, continuity equation of the carrier and the particle phase are used to obtain normal velocity components $V$ and $V_{p}$ respectively by using the following discretization:

$$
\begin{gathered}
V_{i, j}=V_{i-1, j}+\frac{2 n}{(n+1)} Y\left(U_{i, j}-U_{i-1, j}\right)-2 \triangle Y U_{i, j}-4 \frac{X \triangle Y}{\triangle X}\left(U_{i, j}-U_{i, j-1}+U_{i-1, j}\right. \\
\left.-U_{i-1, j-1}\right) \\
V_{p_{i, j}}=V_{p_{i-1, j}}+\frac{2 n}{(n+1)} Y\left(U_{p_{i, j}}-U_{p_{i-1, j}}\right)-2 \triangle Y U_{p_{i, j}}-4 \frac{X \triangle Y}{\triangle X}\left(U_{p_{i, j}}-U_{p_{i, j-1}}+U_{p_{i-1, j}}\right. \\
\left.-U_{p_{i-1, j-1}}\right)
\end{gathered}
$$

At present, rectangular computational domain is used with grid point distribution at equal spacing. Additionally, 1501 uniform grid points are employed in normal $Y$ direction as well as in marching $X$ direction. In the program test, a finer axial step size, $\triangle X=$ $X_{i}-X_{i-1}=0.006$, is found to give acceptable accuracy. The computation has been started from $X_{i}=0.01$ and then marched up to $X_{i}=5.0$ by taking uniform grids. By comparing the results for different grid size in $Y$ directions, we reached at the conclusion to choose $\triangle Y=Y_{j}-Y_{j-1}=0.02$ and the value of the boundary layer $Y_{\infty}$ is 35.0 which actually corresponds to the condition $Y \rightarrow \infty$ and it lies very well outside the momentum and thermal boundary layers of the corresponding phase. Implicit finite difference scheme is unconditionally stable and compatible and hence ensures convergence.

In order to investigate the behavior of drag force and heat transfer rate, solutions are presented in the form of shear stress rate and Nusselt number respectively. Both physical quantities are significant from scientific and experimental point of view. These quantities can be calculated from the following mathematical relations:

$$
\begin{aligned}
& \tau_{w}=C_{f}\left(G r /(4 X)^{-1}\right)^{-1 / 2(n+1)}=\left(\frac{\partial U}{\partial Y}\right)_{Y=0} \\
& Q=N u\left(G r /(4 X)^{n}\right)^{-1 / 2(n+1)}=-\left(\frac{\partial \Theta}{\partial Y}\right)_{Y=0}
\end{aligned}
$$

Now the numerical results obtained for the key parameters are discussed in the section below.

\section{Results and Discussion}

The prime purpose of present study is to analyze the non-Newtonian natural convection boundary layer flow of two-phase dusty fluid. In the analysis, contaminated fluid is moving along a vertical surface and modified power-law viscosity model is used to elucidate the effects of non-Newtonian behavior. The coupled system of equations for the two phase model obtained through primitive variable formulations are solved numerically by the twopoint implicit finite difference method. In order to gain some understanding of this dusty power-law fluid problem along a heated vertical wall, the influence of several important parameters on rate of heat transfer and rate of shear stress, velocity and temperature 
profiles and streamlines and isotherms is presented and discussed. In this analysis, the main point of interest is to find the dominant influence of Prandtl number, Pr, powerlaw index, $n$, mass concentration parameter, $D_{\rho}$, specific heat ratio parameter, $\gamma$ and the dust parameter, $\alpha_{d}$, against the crosswise coordinate $X$. The present numerical solutions are performed for dusty oil (i.e, $\operatorname{Pr}=1000.0, D_{\rho}=10.0, \gamma=0.3$ and $n=1.5$ ). The parametric values for dust parameters for oil are taken from study of Apazidis [36], whereas the values of power-law index $n$ is taken from the analysis of Kumari et al. [12]. During the computations, the overall range of the parameters is set as: $n=1.0,1.2,1.5,1.8,2.0$, $\operatorname{Pr}=500.0,1000.0, \gamma=0.3,0.6,0.9$ and $\alpha_{d}=5.0,10.0,50.0,100.0$.

In order to validate the accuracy of our scheme and numerical computations, comparison is also being made with already available data in literature. It is rather important to mention here that for clear fluid, i.e, $D_{\rho}=\alpha_{d}=0.0$, a number of investigations can be retrieved that are already discussed by various authors and they becomes the special case of the present analysis. For instance, the comparison of rate of heat transfer for different values of Prandtl number is displayed in Tab. 1 for the case of pure Newtonian fluid, i.e., when $D_{\rho}=\alpha_{d}=0.0$ and $n=1$. Clearly, it is seen from Tab. 1 that the computational data of the present problem is in excellent agreement with those of [4], [6], [10]-[12]. When comparing with [11] we have re-scaled rate of heat transfer $Q$ by dividing it with the factor $4^{1 / 4}$. The small difference in numerical values may be due to the use of different methodologies for obtaining the solutions. Besides this, another comparison is also presented in tabular form, with the study of Kumari et al. [12] for the single-phase problem (see Tab. 2). In reference [12], stream function formulations have been used and solutions are presented via Keller box method, while on the other hand, present authors adopted the primitive variable formulation and solved the problem via Thomas algorithm. It can be seen from table that the computational values show good comparison between the studies and ultimately ensures the convergence of our numerical scheme. In Tab. 2, values of rate of heat transfer for i) Newtonian fluid $(n=1)$ and ii) dilatant fluids $(n>1)$ are displayed against the wide range of Prandtl number Pr starting from 0.7 to 1000.0. It is concluded from Tab. 2, that the rate of heat transfer increases sufficiently when both $\operatorname{Pr}$ and $n$ are increased and also the dilatant fluids $(n>1)$ have higher values as compared to the Newtonian fluids $(n=1)$ (see Ref. [12]).

Table 1: Comparison of numerical values of $N u\left(G r /(4 X)^{n}\right)^{-1 / 2(n+1)}$, for $n=1$ (Newtonian fluid), while $\operatorname{Pr}=10.0,100.0,1000.0, D_{\rho}=\alpha_{d}=0.0$ and $\gamma=1.0$.

\begin{tabular}{|c|c|c|c|c|c|c|}
\hline $\operatorname{Pr}$ & $\begin{array}{c}\text { Acrivos } \\
{[4]}\end{array}$ & $\begin{array}{c}\text { EDE } \\
{[6]}\end{array}$ & $\begin{array}{c}\text { Kawase and } \\
\text { Ulbrecht }[10]\end{array}$ & $\begin{array}{c}\text { Huang et al. } \\
{[11]}\end{array}$ & $\begin{array}{c}\text { Kumari et al. } \\
{[12]}\end{array}$ & Present \\
\hline 10 & 0.8943 & 0.8269 & 0.8936 & 0.8268 & 0.82609 & 0.82647 \\
100 & 1.5903 & 1.5506 & 1.5891 & 1.5486 & 1.54739 & 1.54846 \\
1000 & 2.8285 & 2.8047 & 2.8264 & 2.8084 & 2.80689 & 2.80830 \\
\hline
\end{tabular}

The variation of the shear stress rate, $\tau_{w}$ and rate of heat transfer $Q$ are plotted in Fig. 1 for some values of power-law index $n$ and Prandtl number Pr. It can be seen from Fig. 1(a), that the shear stress rate increases sufficiently when $n$ increases from 1.0 to 1.5. This behavior of shear stress rate is quite expected because the shear thickening fluids $(n>1)$ are more viscous as compared to the Newtonian fluid $(n=1)$. Therefore large 
Table 2: Comparison of numerical values of $N u\left(G r /(4 X)^{n}\right)^{-1 / 2(n+1)}$, while $\operatorname{Pr}=$ 0.7,6.7, 10.0, 100.0, 1000.0, $n=1.0,1.2,1.5, D_{\rho}=\alpha_{d}=0.0$ and $\gamma=1.0$.

\begin{tabular}{|c|c|c|c|}
\hline Pr & $n$ & $\begin{array}{c}\text { Kumari et al. } \\
{[12]}\end{array}$ & Present \\
\hline \multirow{3}{*}{0.7} & 1.0 & 0.49841 & 0.49943 \\
& 1.2 & 0.52334 & 0.53108 \\
& 1.5 & 0.55455 & 0.56950 \\
\hline \multirow{3}{*}{6.7} & 1.0 & 1.03974 & 1.04042 \\
& 1.2 & 1.11245 & 1.11286 \\
& 1.5 & 1.20304 & 1.20752 \\
\hline \multirow{3}{*}{10.0} & 1.0 & 1.16891 & 1.16881 \\
& 1.2 & 1.25554 & 1.25634 \\
& 1.5 & 1.36409 & 1.36904 \\
\hline \multirow{3}{*}{100.0} & 1.0 & 2.21251 & 2.21559 \\
& 1.2 & 2.43543 & 2.43349 \\
& 1.5 & 2.71709 & 2.71740 \\
\hline \multirow{3}{*}{1000.0} & 1.0 & 4.13570 & 4.13357 \\
& 1.2 & 4.66234 & 4.66270 \\
& 1.5 & 5.30515 & 5.30278 \\
\hline
\end{tabular}

values of $n$ multiply the viscous effects, which ultimately increases the frictional forces and as a result the drag force at the surface increases. It is observed form Fig. 1(b) that the rate of heat transfer increases as $n$ increases. Besides, it is interesting to see that Prandtl number Pr has also a notable effect on these physical quantities. It is already observed in Tab. 2 that the rate of heat transfer exhibits the tendency to increase for both $n$ and Pr and here again this fact is validated graphically. The shear stress rate decreases for large value of Prandtl number Pr, whereas, the rate of heat transfer get intensified when Pr increases from 500.0 to 1000.0. The Newtonian fluids $(n=1.0)$ have small rate of heat transfer as compared to the dilatant fluids $(n>1)$.

The influence of the dust parameter, $\alpha_{d}$, for oil particulate suspension on $\tau_{w}$ and $Q$, is depicted in Fig. 2. Here, the rate of shear stress and rate of heat transfer are plotted for shear thickening oil for different values of dust parameter $\alpha_{d}=5.0,10.0,50.0,100.0$. It is interesting to infer from Fig. 2(a) that the value of shear stress rate, $\tau_{w}$, increases sufficiently owing to the increase in value of dust parameter, $\alpha_{d}$. Particulary, $\tau_{w}$ is very high for large values of $\alpha_{d}$. The presence of inert particles in oil are responsible for the enhancement of the shear stress rate. As presence of small number of dust particles causes less resistance to the flow, therefore, $\tau_{w}$ is not very influential when the value of dust parameter is small. But an increment in the value of dust parameter offers more resistance to the flow of oil particulate suspension near the leading edge and as a result the frictional forces get strong in the momentum boundary layer region. However, the dust parameter $\alpha_{d}$ a has reverse effect on the rate of heat transfer, $Q$ (see Fig. 2(b)). This may happens due to the fact that, by loading the large number of dust particles, the oil mixture loses the thermal energy and this leads to decrease the temperature of contaminated oil as compared to the pure fluid case. The dilatant (shear thickening) oil becomes more viscous when it is loaded by 
large number of dust particles and ultimately the rate of heat transfer shows a considerable decline. Therefore, it happened subject to the interaction of the two phase flow.

Fig. 3 displays the variation in quantities: $\tau_{w}$ and $Q$ that are brought by changing the value of specific heat ratio parameter $(\gamma)$ of the oil particulate suspension. The shear stress rate shows a considerable decline by increasing the value of $\gamma$ from 0.3 to 0.9 (See Fig. 3(a)). Particulary, the shear stress rate becomes too low when $\gamma=0.9$. The specific heat ratio parameter, therefore, acts a retarding force for the shear stress rate. But on the other hand, it can be visualized from Fig. 3(b) that an opposite behavior is recorded for the rate of heat transfer $Q$. The rate of heat transfer increases sufficiently by magnifying the value of specific heat ratio parameter $(\gamma)$. It can be noted that low specific heat ratio causes a relatively large increase in temperature gradient as compared to large $\gamma$. Interestingly, $\gamma<<1.0$ reflects the physical situation where the specific heat of the oil at constant pressure is less than that of the specific heat of the particle phase, i.e., $\left(c_{s}<c_{p}\right)$, due to which the oil particulate suspension gains more thermal energy which give rise to the temperature gradient and hence assist the rate of heat transfer to enhance.

Fig. 4 is plotted to visualize the variations in the velocity and temperature profiles of oil particulate suspension with the power-law index $n$. The velocity and temperature profiles are recorded at $X=5.0$. It can be seen from Fig. 4(a) that the velocity profile increases sufficiently by increasing the value of power-law index $n$. These plots depicts the fact that for shear-thickening fluids $n=1.2,1.5,1.8,2.0$, the velocity increases and the boundary layer thickness reduces. It is interesting to see that the oil particulate suspension quickly attains its asymptotic value for large values of power-law index $n$. It can be therefore concluded that the non-Newtonian (i.e, $n>1$ or dilatant fluids) are more likely to achieve the limiting behavior as compared to the Newtonian fluids $(n=1.0)$. Fig 4(b) further depict the corresponding temperature distributions of contaminated oil for $1.0 \leq n \leq 2.0$. It is visualized that the temperature profile, $\Theta$, exhibits a decline for increasing values of power-law index $n$ and dilatant fluids $(n>1)$ quickly attains the limiting value into the thermal boundary layer region. Such behavior reveals the fact that temperature profile is high for the Newtonian fluids $(n=1)$ than the dilatant fluids $(n>1)$.

Streamlines and isotherms are also drawn in Fig. 5 for oil particulate suspension. These plots help in accessing the performance of the flow velocity and temperature fields of dusty power-law fluid moving along a heated isothermal vertical wall. The effect of mass concentration parameter $D_{\rho}$ on the distribution of the velocity and temperature fields is plotted for Newtonian (i.e, $n=1.0$ ) and non-Newtonian shear thickening (i.e, $n>1$ ) fluids. For comparison, oil suspension with small number of particle cloud $\left(D_{\rho}=10.0\right)$ is also presented. As expected, by increasing the particle in the fluid, the velocity of dusty fluid reduces significantly and fluid tends to cluster near the vicinity of the vertical surface (see Figs. 5(a) and 5(b)). More interestingly, the influence of $D_{\rho}$ on temperature distribution for both phases is notable. When particles are loaded extensively, the relative velocity between the two phases reduces and relaxation time for energy transfer also decreases and ultimately temperature lags between the mixture components become smaller. The thermal boundary layer becomes thinner due to the large temperature lags between the phases (see Figs. 5(c) and $5(\mathrm{~d})$ ). Furthermore, the isotherms in Fig. 5 is revealing the fact that for large values of mass concentration parameter $D_{\rho}$ curves are very close to each other for Newtonian (i.e, $n=1.0$ ) as well as for non-Newtonian shear thickening (i.e, $n>1$ ) fluids. 


\section{Conclusions}

The present analysis aims to compute the numerical results for two-phase dusty boundarylayer flow induced by the semi-infinite vertical heated surface. In order to incorporate the non-Newtonian fluid into the analysis, modified viscosity model is used. Coordinate transformations (primitive variable formulations) are applied to switch the governing equations of the carrier and the dispersed phase into a convenient form over which two point finite difference method is applied to obtain the solutions for the whole range of axial coordinate $X$. Computational results are shown for the physical quantities, namely, rate of shear stress, rate of heat transfer, velocity and temperature profiles, streamlines and isotherms. Results are interpreted by considering contaminated oil as a working fluid, which is under the influence of several physically important parameters, such as, $n, \operatorname{Pr}, D_{\rho}, \alpha_{d}$ and $\gamma$. The agreement with the results of [4], [6], [10]-[12] are found excellent. It is recorded that, the Newtonian fluids $(n=1.0)$ have small rate of heat transfer as compared to the dilatant fluids $(n>1)$ and the rate of heat transfer deliberately boosted by increasing the values of power-law index $n$ and Prandtl number Pr. It is also found that the shear stress rate increases when the dust parameter $\alpha_{d}$ increases and the rate of heat transfer drastically decreases when metal particles are increased within the oil. In addition, the temperature profile, $\Theta$, for dilatant fluids $(n>1)$ quickly attains the limiting value into the thermal boundary layer region as compared to the Newtonian fluid $(n=1.0)$.

\section{References}

[1] Ostwald, W., Ueber die rechnerische Darstellung des Strukturgebietes der Viskositt, Kolloid Z., 47, 1929, 176-187.

[2] Schowalter, W. R., The application of boundary layer theory to power law pseudoplastic fluids, AIChE J., 6, 1960, 24-28.

[3] Lee, S. Y., Ames, W. F., Similar solutions for non-Newtonian fluids, AIChE., 12, 1966, 700-708.

[4] Acrivos, A., A theoretical analysis of laminar natural convection heat transfer to non-Newtonian fluids, AIChE J., 16, 1960, 584-590.

[5] Na, T. Y., Hansen, A. G., Possible similarity solutions of the laminar natural convection flow of non-Newtonian fluids, Int. J. Heat Mass Transf., 9, 1966, 261-262.

[6] EDE, A. J., Advances in free convection, Advances in Heat Transf., 4, 1967, 1-64, Academic, New York.

[7] Sharma, K. K., Adelman, M., Experimental study of natural convection heat transfer from a vertical plate in a non-Newtonian fluid, Can. J. Chem. Eng., 47, 1969, 553-555.

[8] Dale, J. D., Emery, A. F., The free convection of heat from a vertical plate to several non-Newtonian pseudoplastic fluids, J. Heat Transf., 94, 1972, 64-72.

[9] Chen, T. V. W., Wollersheim, D. E., Free convection at a vertical plate with uniform flux condition in non-Newtonian power-law fluids, J. Heat Transf., 95, 1973, 123-124. 
[10] Kawase, K., Ulbrecht, J., Approximate solution to the natural convection heat transfer from a vertical plate, Int. Commun. Heat Mass Transf., 11, 1984, 143-155.

[11] Huang, M. J., Huang, J. S., Chou, Y. L., Chen, C. K., Effects of Prandtl number on free-convection heat transfer from a vertical plate to a non-Newtonian fluid, J. Heat Transf., 111, 1989, 189-191.

[12] Kumari, M., Pop, I., Takhar, H. S., Free convection boundary layer flow of a nonNewtonian fluid along a vertical wavy surface, Int. J. Heat and Fluid Flow, 18, 1997, 625-631.

[13] Pop, I. Gorla, R. S. R., Mixed convection similarity solutions of a non-Newtonian fluid on a horizontal surface, J. Heat Mass Transf., 33, 1991, 57-63.

[14] Wang, T. Y., Mixed convention heat transfer from a vertical plate to non-Newtonian Fluids, Int. Commun. Heat Mass Transf., 22, 1995, 212-219.

[15] Denier, J. P., Hewitt, R. E., Asymptotic matching constraints for a boundary-layer flow of a power-law fluid, J. Fluid Mech., 518, 2004, 261-279.

[16] Hossain, M. A., Grola, R. S.R., Natural convection flow of non-Newtonian power-law fluid from a slotted vertical isothermal surface, Int. J. Numer. Method H., 19, 2009, 835-846.

[17] Molla, M. M., Yao, L. S., The flow of non-Newtonian fluids on a flat plate with a uniform heat flux, ASME J. Heat Transf., 131, 2009, 1-6.

[18] Rudinger, G., Fundamentals of gas-particle flow, Elsevier Scientific Publishing Co., Amsterdam, 1980.

[19] Farbar, L., Morley, M. J., Heat transfer to flowing gas-solid mixtures in a circular tube, Ind. Eng. Chem., 49, 1957, 1143-1150.

[20] Marble, F. E., Dynamics of a gas containing small solid particles, combustion and propulsion, 5th AGARD colloquium, Pergamon press, 1963.

[21] Singleton, R. E., Fluid mechanics of gas-solid particle flow in boundary layers, Ph.D. Thesis, California Institute of Technology, 1964.

[22] Michael, D. H., Miller, D. A., Plane parallel flow of a dusty gas, Mathematica, 13, 1966, 97-109.

[23] Saffman, P. G., On the stability of laminar flow of a dusty gas, J. Fluid Mech., 13, 1962, 120-128.

[24] Michael, D. H., The steady motion of a sphere in a dusty gas, J. Fluid Mech., 31, 1968, 175-192.

[25] Datta, N., Mishra, S. K., Boundary layer flow of a dusty fluid over a semi-infinite flat plate, Acta Mech., 42, 1982, 71-83. 
[26] Agranat, V. M., Effect of pressure gradient of friction and heat transfer in a dusty boundary layer, Fluid Dyn., 23, 1988, 729-732.

[27] Gireesha, B. J., Bagewadi, C. S., Prasannakumara, B. C., Pulsatile flow of an unsteady dusty fluid through rectangular channel, Commun. Nonlinear Sci. Numer. Simulat., 14, 2009, 2103-2110.

[28] Roopa, G. S., Gireesha, B. J., Bagewadi, C. S., Numerical investigation of mixed convection boundary layer flow of a dusty fluid over an vertical surface with radiation, Afr. Math., 24, 2013, 487-502.

[29] Manjunatha, P. T., Gireesha, B. J., Prasannakumara, B. C., Thermal analysis of conducting dusty fluid flow in a porous medium over a stretching cylinder in the presence of non-uniform source/sink, Int. J. Mech. Mater. Eng., 9, 2014, doi:10.1186/s40712014-0013-8.

[30] Siddiqa, S., Hossain, M. A., Saha, S. C., Two-phase natural convection flow of a dusty fluid, Int. J. Numer. Method, 25, 2015, 1542-1556.

[31] Prasannakumara, B. C., Gireesha, B. J., Manjunatha, P. T., Melting phenomenon in MHD stagnation point flow of dusty fluid over a stretching sheet in the presence of thermal radiation and non-uniform heat source/sink, Int. J. Comp. Methods Eng. Sci. Mech., 16, 2015, 265-274.

[32] Siddiqa, S., Begum, N., Hossain, M. A., Massarotti, N., Influence of thermal radiation on contaminated air and water flow past a vertical wavy frustum of a cone, Int. Commun. Heat Mass Transf., 76, 2016, 63-68.

[33] Siddiqa, S., Begum, N., Hossain, M. A., compressible dusty gas along a vertical wavy surface, Appl. Math. Comput., 293, 2017, 600-610.

[34] Chamkha, A. J., Unsteady flow of a power-law dusty fluid with suction, ASME J. Fluids Eng., 115, 1993, 330-333.

[35] Siddiqa, S., Begum, N., Hossain, M. A., Radiation effects from an isothermal vertical wavy cone with variable fluid properties, Appl. Math. Comput., 289, 2016, 149-158.

[36] Apazidis, N., Temperature distribution and heat transfer in a particle-fluid flow past a heated horizontal plate, Int. J. Multiphase Flow, 16, 1990, 495-513. 

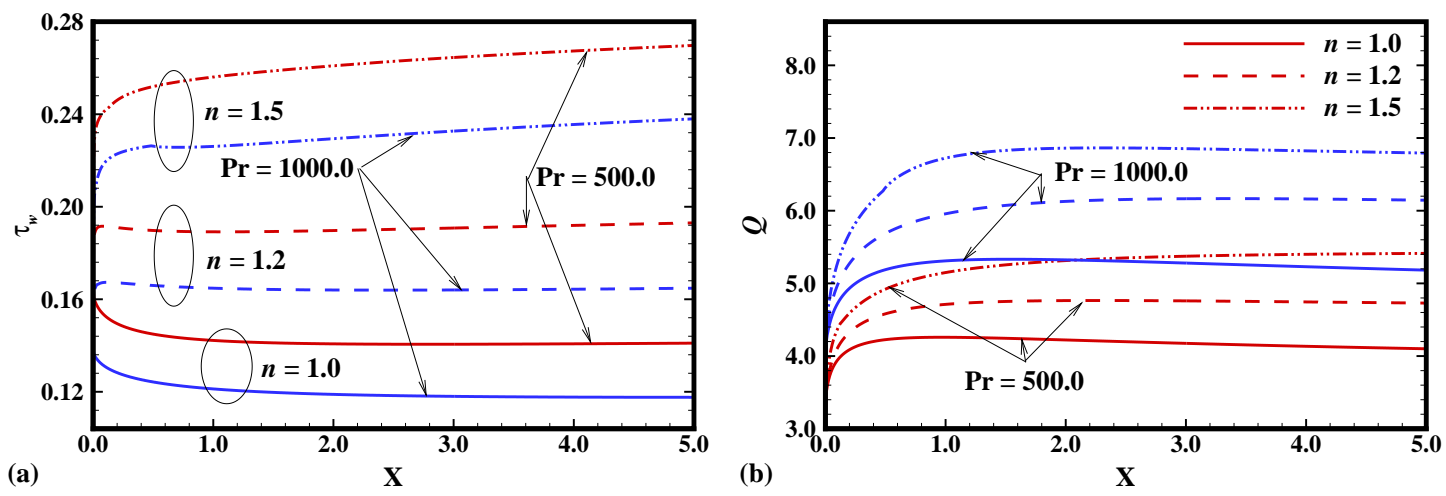

Fig. 1(a) Shear stress and (b) Rate of heat transfer for $n=1.0,1.2,1.5$, $\operatorname{Pr}=$ 500.0, 1000.0, $\alpha_{d}=5.0, D_{\rho}=10.0, \gamma=0.3$.
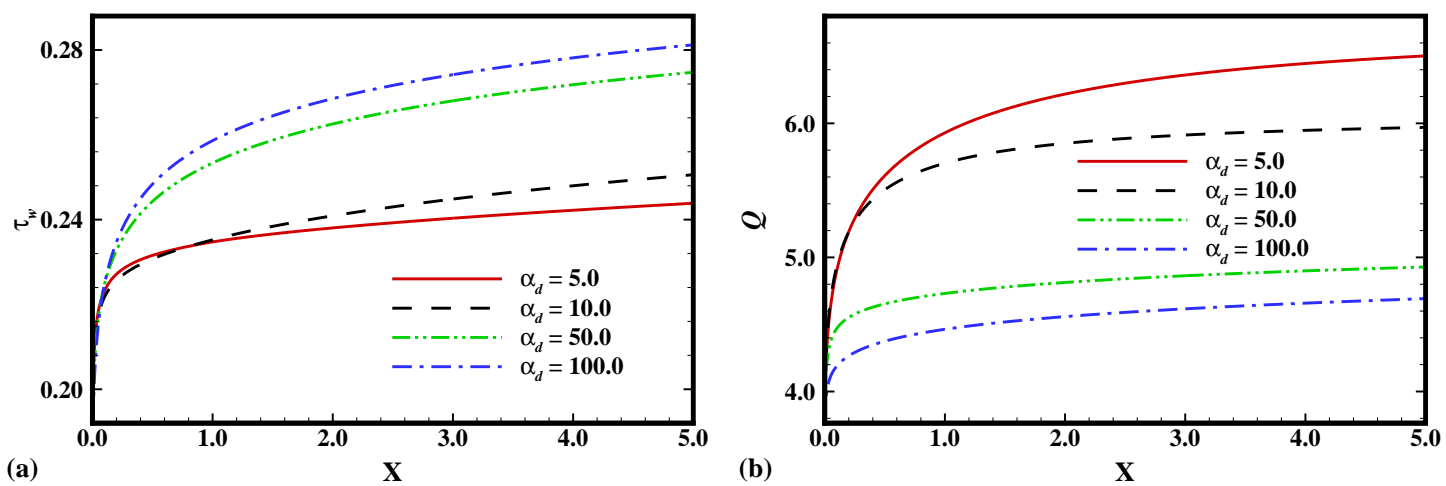

Fig. 2(a) Shear stress and (b) Rate of heat transfer for $\alpha_{d}=5.0,10.0,50.0,100.0$, $D_{\rho}=10.0, \gamma=0.3, \operatorname{Pr}=1000.0, n=1.5$.
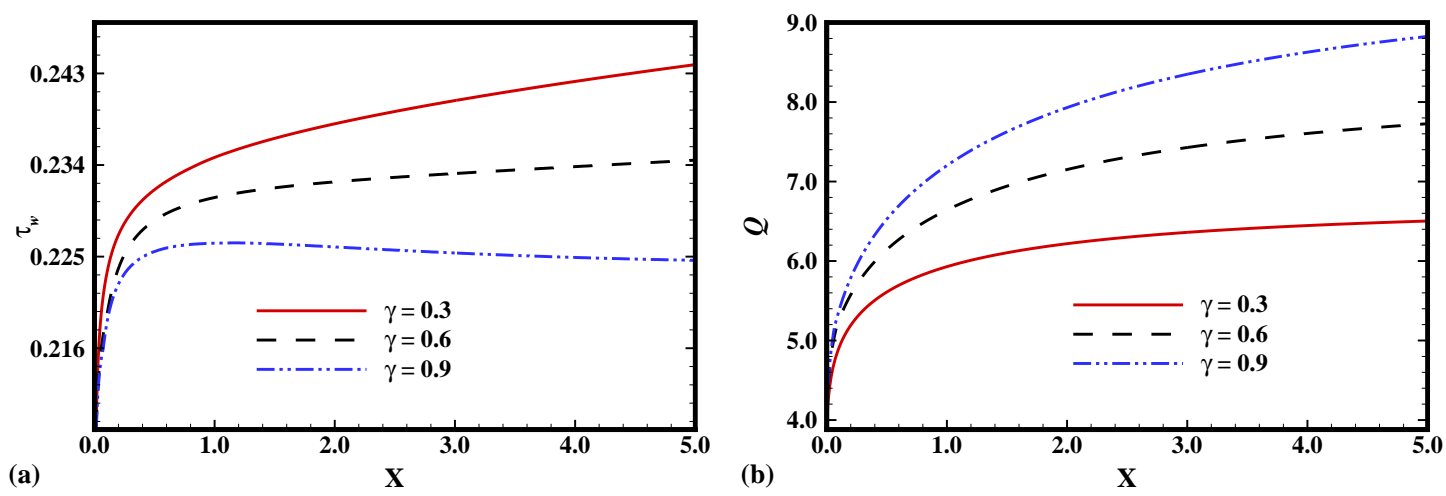

Fig. 3(a) Shear stress and (b) Rate of heat transfer for $\gamma=0.3,0.6,0.9, \alpha_{d}=5.0$, $D_{\rho}=10.0, \operatorname{Pr}=1000.0, n=1.5$. 

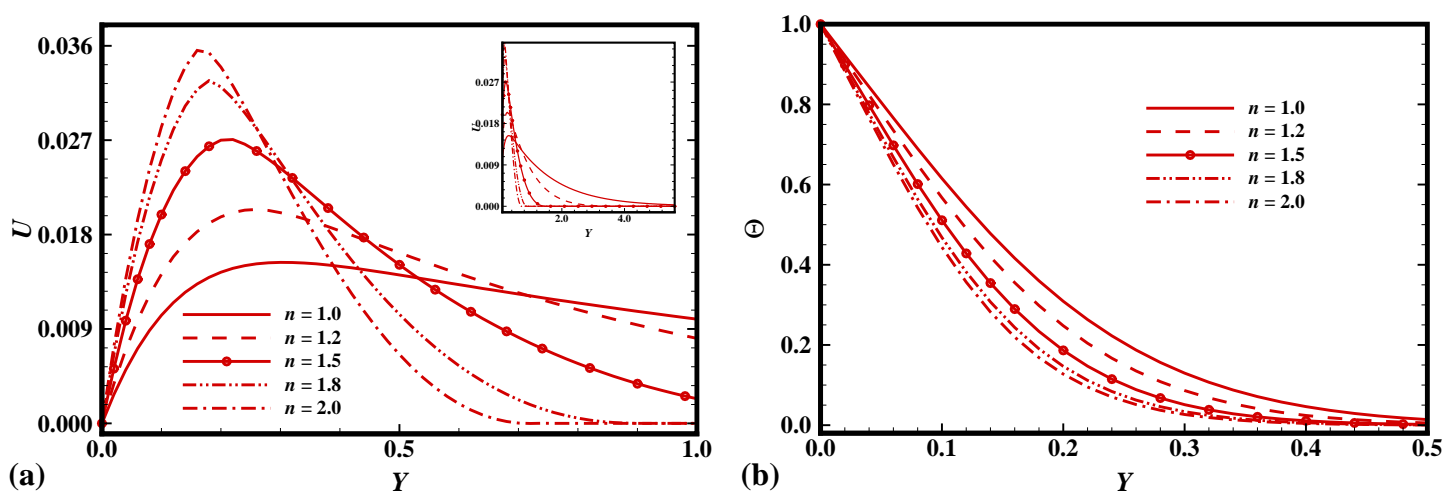

Fig. 4(a) Velocity and (b) Temperature profiles of the carrier phase for $n=1.0,1.2,1.5,1.8,2.0, D_{\rho}=10.0, \operatorname{Pr}=500.0, \gamma=0.3, \alpha_{d}=5.0, X=5.0$.
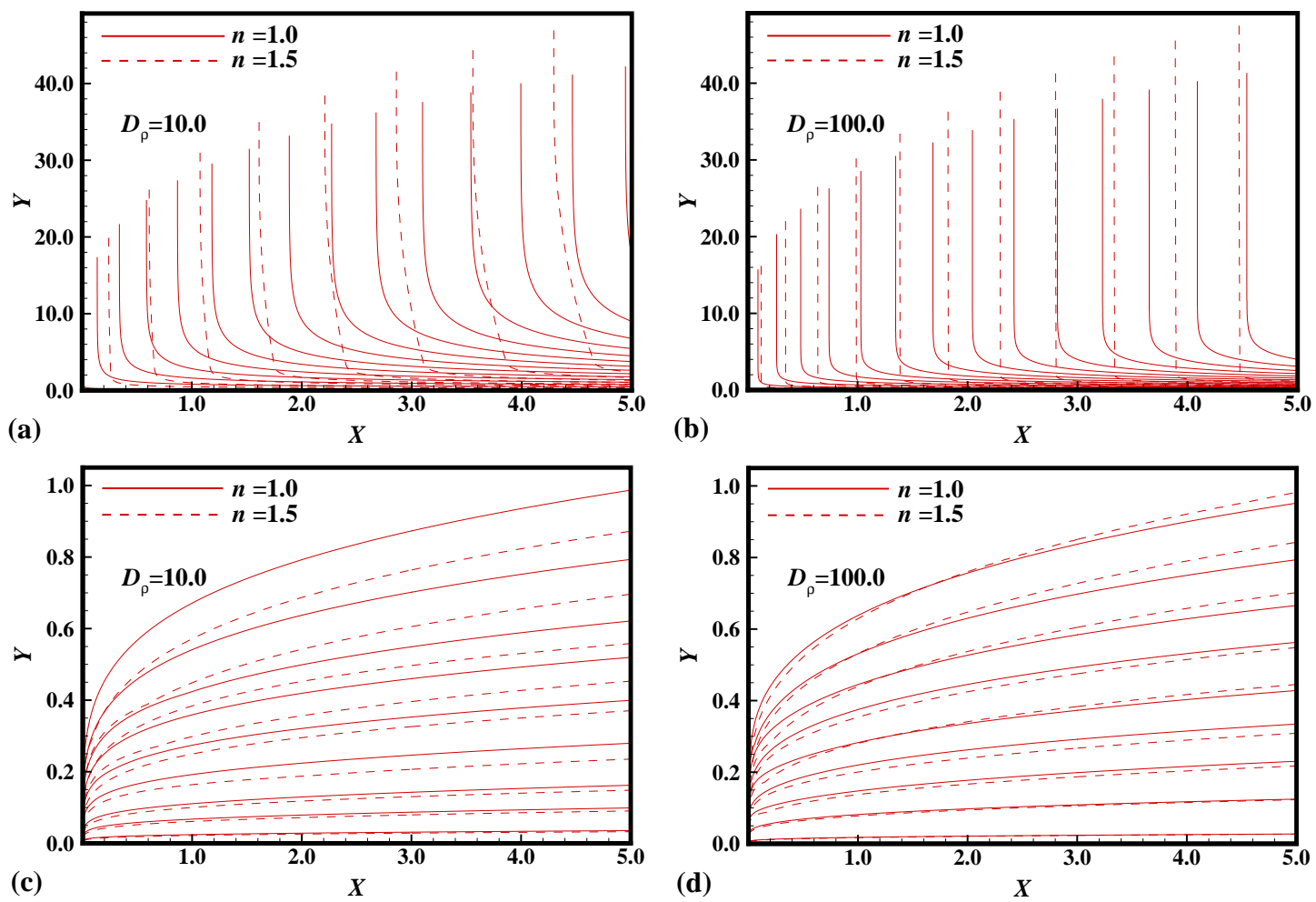

Fig. 5 Streamlines (top) and Isotherms (bottom) of the carrier phase for $D_{\rho}=10.0,100.0, \alpha_{d}=5.0, \gamma=0.3, \operatorname{Pr}=1000.0, n=1.5$. 